\title{
Etiology of cutaneous vasculitis: utility of a systemic approach
}

\author{
Caroline Chanussot-Deprez1, María Elisa Vega-Memije², Luis Flores-Suárez³, Celia Ríos-Romero4, \\ Javier Cabiedes-Contreras ${ }^{3+}$, Edgardo Reyes ${ }^{5}$ and Lucia Rangel-Gamboa ${ }^{2}$ \\ ${ }^{1}$ Dermatology Department, Hospital General-PEMEX, Veracruz, Ver.; ${ }^{2 B}$ Biomedical Research Deputy Director's Office, Hospital General Dr. Manuel \\ Gea González, Ciudad de México; ${ }^{3}$ Department of Immunology and Rheumatology, Instituto Nacional de Ciencias Médicas y Nutrición, Ciudad de
} México; ${ }^{4}$ Private practice, Querétaro, Qro.; ${ }^{5}$ Pathology Department, Instituto Nacional de Ciencias Médicas y Nutrición, Ciudad de México, Mexico

\begin{abstract}
Cutaneous vasculities (CV) represents a diagnostic challenge, occurs as primary cutaneous disorder or as a manifestation of other entities. Objective: To search the cause of CV. Methods: Patients with CV were prospectively evaluated. In all patients, skin biopsies were drawn, and direct immunofluorescence was done in most of the patients. American College of Rheumatology (ACR) and Chapel Hill Consensus Conference Criteria (CHCC) were used for classification. Results: 32 patients were studied. There was female predominance (71.8\%). Children presented drug-associated CV or Schönlein-Henoch purpura(SHP). Adults presented more frequently SHP, systemic lupus erythematosus or paraneoplastic vasculitis, other diagnosis as polyarteritis nodosa, microscopic polyangiitis, thrombotic vasculitis (post-puerperal), antiphospholipid syndrome, Churg-Strauss syndrome, and drug-associated CV were presented. Using the ACR and CHCC criteria, 50\% of cases were classified. Discussion: In our institution, during this work the etiologic diagnostic of $\mathrm{CV}$ increased more than twice. However, in the case of HSV and SHP none of the proposed criteria had high specificity; other parameters were used to discern between both. Six patients remained as not classified. In our view, cryoglobulins and hepatitis serology do not seem useful unless patient's history supports they need to be done. Unclassified patients were followed-up closely for 2 years.
\end{abstract}

KEY WORDS: Cutaneous vasculities. Vasculitis. Purpura. Lupus. Rheumatic disease.

\section{Introduction}

Vasculitis is a clinicopathological process characterized by blood vessel inflammation and necrosis, which entails their occlusion and causes tissue ischemia $^{1,2}$. Cutaneous vasculitis (CV) etiologic diagnosis constitutes a challenge, since it can be primary or early manifestations of different systemic diseases such as systemic necrotizing vasculitis, connective tissue diseases (systemic lupus erythematosus [SLE], systemic infections or neoplasms ${ }^{3-5}$. The skin offers an opportunity for diagnosis, since it is an accessible source for anatomopathological study ${ }^{6}$.
CVs are classified based on clinical and histological data using the American College of Rheumatology (ACR) criteria since $1990^{7}$ and the Chapel Hill Consensus Conference (CHCC) criteria issued in 1992 (revised in 2012); ; although these classifications are not entirely satisfactory, they continue to be used in clinical practice ${ }^{9}$. Vasculitides are grouped according to the affected vessel size as: a) large vessel vasculitis, which includes giant cell arteritis and Takayasu $\operatorname{arteritis}^{10}$; b) medium vessel vasculitis, such as polyarteritis nodosa (PAN), cutaneous PAN and Kawasaki's disease ${ }^{11}$; and c) small vessel vasculitis, which can be granulomatous such as granulomatosis with

\author{
Correspondence: \\ Lucía Rangel-Gamboa \\ Subdirección de Investigación \\ Hospital General Dr. Manuel Gea González \\ Calzada de Tlalpan 4800 \\ Col. Belisario Domínguez Sección XVI, Del. Tlalpan \\ C.P. 14080 , Ciudad de México, México \\ E-mail: draluciarangel@yahoo.com.mx
}

Date of reception: 19-12-2016

Date of acceptance: $30-12-2016$

DOI://dx.doi.org/10.24875/GMM.M18000113
Gac Med Mex. 2018;154:49-54

Contents available at PubMed www.gacetamedicademexico.com 
polyangiitis, formerly known as Wegener granulomatosis ${ }^{12}$, and eosinophilia with polyangiitis, also known as Churg-Strauss Syndrome (SCS) ${ }^{13}$; and non-granulomatous, which include microscopic polyangiitis $(\mathrm{MPA})^{14}$, Schönlein-Henoch purpura (SHP) $)^{15}$, cutaneous leukocytoclastic vasculitis (LCV) ${ }^{16}$ and essential cryoglobulinemia ${ }^{17}$.

Vasculitis etiologic factors are diverse and include infectious agents (15-20\%), inflammatory conditions (15-20\%), medications (10-15\%) and neoplasms (5\%), with $45-55 \%$ of cases not having an etiologic diagnosis $^{5,18-20}$.In tertiary care referral centers, SLE-associated vasculitides occur more often in adult patients ${ }^{21}$. Vasculitis clinical diagnosis must be confirmed by anatomopathological study, where loss of vascular architecture is observed, caused by wall infiltration by neutrophils, lymphocytes or histiocytes; the presence of fibrinoid necrosis is characteristic ${ }^{22}$. Direct immunofluorescence can be positive in blood vessels in specific etiologies the direct immunofluorescence can be positive in blood vessels and is useful to classify and confirm the presence of vasculitis and to identify the type of immunoglobulin (Ig) deposited on the vascular wall ${ }^{23}$; for example, the presence of $\lg \mathrm{A}$ is associated with SHP, while IgM can be observed in cryoglobulinemic vasculitis.

CVs account for $0.13 \%$ of outpatient consultations at Dermatology department of General Hospital "Dr. Manuel Gea González". However, there was no diagnostic protocol for these patients focused on determining the etiology. In previous years, etiology was diagnosed in $25 \%$ of cases. Therefore, the purpose of this work was to define CV etiologic diagnosis in a general hospital during a 2-year period, and to increase the percentage of etiologic diagnosis. In this study, both previously-mentioned classifications were applied (Table 1) $)^{24,25}$

\section{Method}

Children and adults with clinical and anatomopathological diagnosis of $\mathrm{CV}$, who attend to dermatology department of General Hospital "Dr. Manuel Gea González" during a 2-year period were included. To be incorporate in the study, patients or their legal guardians signed an informed consent form. The study protocol was approved by the institutional ethics and research committee, and for the performance of biopsy and sample taking, the principles of the Declaration of Helsinki were followed. Patients were prospectively studied, with assessment including a detailed clinical
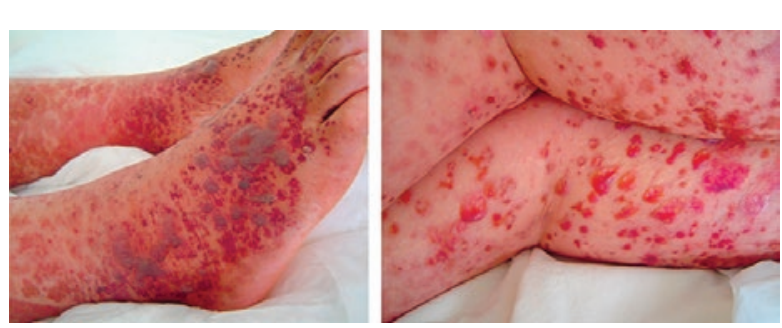

Figure 1. Palpable purpura in an adult patient diagnosed with Schönlein-Henoch purpura.

evaluation and physical examination. Two samples of the skin lesions were obtained by means of a $4-\mathrm{mm}$ punch biopsy technique, one for staining with hematoxylin and eosin and another for direct immunofluorescence (DIF). Complementary tests were requested, including: blood count, erythrocyte sedimentation rate, blood chemistry, general urine test, fecal occult blood (stool guaiac test), serum creatinine, coagulation times, total immunoglobulin (IgA, IgG, IgM), rheumatoid factor, anti-nuclear auto-antibodies (ANA), anti-neutrophil cytoplasm antibodies (ANCA), anti-myeloperoxidase (MPO) and anti-proteinase 3 (PR-3), cryoglobulins, anti-phospholipids, IgG, IgA, IgM, C3 and C4 complement, as well as serology for hepatitis $B$ and $C$. The $A C R$ and $\mathrm{CHCC}$ criteria were used to define etiologic diagnosis. Final operational diagnosis was established by consensus between observers at the conclusion of the diagnostic protocol.

Forty-four patients with clinical diagnosis of CV were initially included in the protocol, out of which 12 were eliminated: in 8 patients, vasculitis was not found by histopathologic study, two pediatric patients were diagnosed with thrombocytopenic purpura, other patient had systemic large vessel vasculitis without CV and, finally, one patient refused to participate in the protocol.

\section{Results}

Of a total of 44 patients with clinical diagnosis of vasculitis, 5 children and 27 adults were included. Predominance was observed in the female gender $(71.8 \%$ vs. $28.2 \%)$. The mean age at diagnosis was 37.75 years (range: 6-73 years). Children were 6, 8, 13 (2 patients) and 17 years' old. Clinical manifestations predominated in the lower limbs $(69 \%)$ and the trunk, with varied morphology, and with palpable purpura $(69 \%)$ and erythematopurpuric maculae $(60 \%)$ being more common (Fig. 1). Some patients had ulcers $(16 \%)$ and nudosities (6\%). 
Table 1. Vasculitis name and definition adopted by the CHCC and the ACR (modified from Carlson et al. 2005)

\begin{tabular}{ll}
\hline Large vessel vasculitis & Chapel Hill Consensus Conference \\
Temporal arteritis & $\begin{array}{l}\text { Granulomatous arteritis of the aorta or its major } \\
\text { branches (intracranial) } \\
\text { Often involves the temporal artery } \\
\text { Patients older than } 50 \text { years }\end{array}$ \\
Takayasu arteritis & $\begin{array}{l}\text { Granulomatous inflammation of aorta or its major } \\
\text { branches } \\
\text { Usually in patients younger than } 50 \text { years }\end{array}$ \\
Medium vessel vasculitis & \\
Polyarteritis nodosa & $\begin{array}{l}\text { Necrotizing inflammation of the medium and small } \\
\text { vessels without glomerulonephritis }\end{array}$ \\
Kawasaki disease & $\begin{array}{l}\text { Asteritis that affects large, medium and small vessels } \\
\text { syndrome }\end{array}$
\end{tabular}

\section{Small vessel vasculitis}

Polyangiitis with granulomatosis (Wegener granulomatosis)

Churg-Strauss syndrome

Microscopic polyangiitis

Granulomatous lesions can affect the respiratory tract, granulomatous vasculitis that affects medium and small caliber vessels

Eosinophil-rich granulomatous inflammation that affects the respiratory tract

Necrotizing vasculitis that affects small and medium vessels and is associated with eosinophilia and asthma

American College of Rheumatology

50 years

Headache

Abnormal temporal artery on physical Elevated erythrocyte sedimentation Temporal biopsy shows vasculitis

Patients younger than 40 years Claudication

Arterial pulse decrease

Blood pressure difference $>10 \mathrm{mmHg}$ between arms

Abnormal arteriogram

Weight loss larger than $4 \mathrm{~kg}$ Livedo reticularis Testicular pain or hypersensitivity Myalgia, myopathy

Neuropathy

Hypertension (diastolic $>90 \mathrm{mmHg}$ ) Kidney involvement Abnormal arteriography Neutrophils observed in biopsy

Necrotizing vasculitis with few or no immune deposits that affects small vessels (capillaries, venules and arterioles)

Glomerulonephritis

Lung involvement

Schönlein-Henoch purpura

Vasculitis with immune deposits, predominantly IgA, affecting small vessels (capillaries, venules and arterioles).

Often affects the skin, intestine, glomerulus and can be associated with arthralgia or arthritis

Cryoglobulinemic vasculitis

Vasculitis with immune cryoglobulin deposits affecting small vessels and that is associated with the presence of serum cryoglobulins Skin and glomeruli are often involved vasculitis)

Nasal or oral inflammation, presence of nodules, infiltrate or cavitations in chest X-ray Microscopic hematuria Granulomatous inflammation in biopsy

Asthma

Eosinophils $>10 \%$

Neuropathy

Pulmonary infiltrates

Extravascular eosinophils in biopsy

Palpable purpura

Patients younger than 20 years Intestinal angina

Neutrophils on vascular wall observed in biopsy

Patients older than 16 years Associated with medications Palpable purpura Rash

Positive biopsy 
SHP was diagnosed in 4 patients, 3 adults and one child (13\%), CV associated with connective tissue diseases occurred in 2 adult patients who were diagnosed with SLE (6\%), whereas vasculitis secondary to medication ingestion or hypersensitivity vasculitis (HSV) occurred in one adult and two children (9\%); 2 cases were associated with neoplasms, which were diagnosed after vasculitis onset. For each one of the next conditions PAN, MPA, anti-phospholiped syndrome (APS), SCS and thormbotic vasculitis) post-puerperal) one patient was diagnosis. Using ACR vasculitis criteria, $44 \%$ of patients were classified and 2 more using ACR criteria for SLE; therefore in 50\% of patients, etiologic diagnosis was identified. The rest did not meet the criteria or there were no criteria for their condition. We found overlapping of SHP and HSV criteria in 12 cases, with other parameters being used to discern between these diagnoses in case of doubt. Of these, $50 \%$ had one or the other pathology, the rest were false positives ( 5 could not be classified, other was diagnosed as paraneoplastic syndrome during follow-up). Applying $\mathrm{CHCC}$ criteria, $50 \%$ of patients were classified without diagnostic overlapping; of the 12 patients with LCV initial diagnosis, in 3 of them was the diagnosis confirmed, with the rest being false positives (one paraneoplasm, 2 SHP and 6 patients could not be classified). No patient had cryoglobulinemia or hepatitis-positive serology for. Finally, 18 patients $(57 \%)$ were classified with the $\mathrm{CHCC}$ criteria during the 2-year follow-up.

\section{Discussion}

In our institution, 12.4 patients are diagnosed with CV per year, which represents $0.13 \%$ of outpatient consultation, according to a review of medical records for a 6 -year period. Previously, the rate of etiologic diagnosis in CV cases was 25\%. In this study, an etiologic diagnosis was established in $57 \%$ of CV cases, which is similar to rates reported in other groups $(51 \%)^{26}$. On initial examination, $56 \%$ of patients were not classified, during de follow up two patients which represents $6 \%$ ) received an etiologic diagnosis, the follow-up was carried out every 6 months during 2 years, which included laboratory tests and biopsy when it was required, which facilitated for an opportune diagnosis to be established and for systemic complications to be avoided.

CV was more common in females (71.8\%), with a mean age of 37.75 years at onset. The most common diagnosis in children was HSV, although SHP is generally considered the most common cause of vasculitis in children; however, in this case series this finding can be explained, since, in our hospital, children with SHP are referred to the pediatrics department, given that digestive, renal and articular symptoms are usually more severe than cutaneous lesions. In adults, the most common cause of CV was SHP, followed by SLE and cases associated with neoplasms. Previous studies reported a series of 160 cases with LCV where $10 \%$ of them were associated with collagen diseases, which was similar to that reported in our patients, while other series report up to $20 \%$. CV occurred as palpable purpura in the lower limbs in $22 / 32$ patients $(68.7 \%)$; in other reports, $51 \%$ of patients with CV show this palpable purpura ${ }^{27}$. Cutaneous ulcers as sole manifestation were found in $15.6 \%$, including patients with the following diagnoses: PAN, APS and SLE, thus we consider that patients with lower limb ulcers who are not responsive to treatment should be studied in order to rule out CV.

In the laboratory tests, serum IgA was elevated in 14 patients; 2 with SHP, one case of drug-associated vasculitis, one with SLE and 10 with other diagnoses. SHP is a systemic vasculitis where IgA plays a preponderant pathophysiologic role and can be found on skin, kidney and intestinal vascular walls ${ }^{28,29}$. However, serum IgA is not always elevated in these patients (only $22 \%$ of cases are positive) and ACR does not consider it to be a diagnostic criterion ${ }^{30}$. Moreover, the presence of IgA on the vascular wall, detected by DIF, is regarded as a diagnostic criterion for SHP by CHCC.

In patients diagnosed with SLE and CV, ANA and the complement were found within normal limits, which can be explained because patients were receiving immunosuppressive treatment for SLE. However, we consider important for these tests to be performed during follow-up, especially in patients with lymphocytic vasculitis ${ }^{30}$. ANCAs constitute an important test in the diagnosis of polyangiitis with granulomatosis, MPA and SCS. p-ANCA and MPO association has $99 \%$ specificity, with C-ANCA and PR-3 being similarly associated ${ }^{31-33}$. Blanco et al. found that $30 \%$ of adult patients with CV had systemic vasculitis or an associated condition (pulmonary or renal involvement or mononeuritis); therefore, it is important for these tests to be carried out searching for the etiology of a $\mathrm{CV}^{5}$. With regard to DIF, performing a biopsy of the lesion is considered more appropriate than in perilesional skin, if tissue necrosis is not observed in the lesion ${ }^{22}$. 


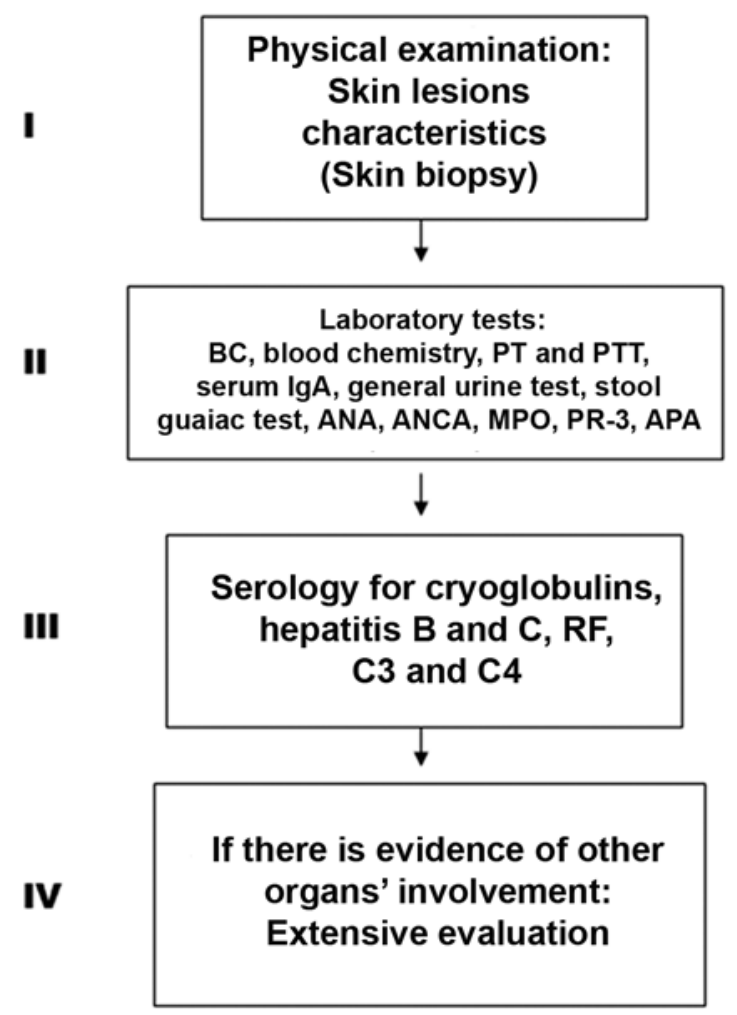

Figure 2. Flow diagram. ANA: anti-nuclear antibodies; ANCA: anti-neutrophil cytoplasmantibodies; APA: antiphospholipid antibodies; $B C$ : blood count; MPO: anti-myeloperoxidase; PR-3: proteinase 3; PT: prothrombin time; PTT: partial thromboplastin time.

The presence of IgA on the vascular wall is not pathognomonic, but it is highly sensitive (82\%) and specific $(73 \%)$ for LCV diagnosis, and it was associated mainly with the presence of SHP ${ }^{34,35}$. Recently, a series of 8 cases was published where presence of $\lg \mathrm{A}$ was found in lymphocytic vasculitis cases that were associated or not with $\mathrm{SLE}^{36}$. The presence of IgM or IgG without IgA does not rule out LCV diagnosis, but it doesn't support it ${ }^{34}$. In our cases we observed IgA on the vascular wall in 4 patients with SHP, while other patients exhibited IgM and $\mathrm{C} 3$ or negative DIF. These results may be explained by IgA lability, which rapidly disappears by proteolysis and phagocytosis in necrotic lesions. The presence of IgA on cutaneous vessels is associated with renal damage, but not with its severity ${ }^{37,38}$.

It is difficult for CV diagnosis to be established by applying the ACR and CHCC criteria, especially in patients with SHP or HSV, as these classifications do not help to differentiate between both these entities. DIF with IgA present on blood vessels can be of help in some cases. This problem is explained because ACR criteria were designed based on series of clinical, laboratory and histopathologic tests that determined the most sensitive and specific characteristics for the different types of vasculitis, but they are not always helpful to differentiate between two diagnostic possibilities.

Evidence in this case series indicates that in the Mexican population, unlike other populations, cryoglobulins and hepatitis $B$ and $C$ are not useful as initial tests, unless patient medical history suggests so.

\section{Conclusions}

ACR criteria are currently not entirely consistent with the definitions of specific conditions and they don't always allow an accurate diagnostic approach. On the other hand, $1994 \mathrm{CHCC}$ provides definitions of the causes of vasculitis and includes the use of some markers when histologic study is not available. CHCC definitions were revised in $2012^{8}$, with the purpose to incorporate new terminologies; however, CHCC delivered a nomenclature rather than a classification. In this context, we consider that inclusion of tests such as ANCA facilitates the diagnostic approach $^{31,32,39}$. According to our findings, we propose the study protocol shown in figure 2 for patients with vasculitis.

\section{References}

1. Gonzalez-Gay MA, Garcia-Porrua C, Pujol RM. Clinical approach to cutaneous vasculitis. Curr Opin Rheumatol. 2005;17(1):56-61.

2. Sneller M, Fauci A. Pathogenesis of vasculitis sindromes. Med Clin North Am. 1997;81(1):221-42.

3. Ramirez GA, Weyand C, Vaglio A, et al. Editorial: Vascular inflammation in systemic autoimmunity. Front Immunol. 2016;21(7):471.

4. Chen KR, Carlson JA. Clinical approach to cutaneous vasculitis. Am J Clin Dermatol. 2008;9(2):71-92.

5. Blanco R, Martínez-Taboada V, Rodríguez-Valverde V. Cutaneous vasculitis in children and adults: associated diseases and etiologic factors in 303 patients. Medicine. 1998;77(6):403-18.

6. Crowson AN, Minm M, Magro C. Cutaneous vasculitis: a Review. J Cutan Pathol. 2003;30(3):161-73.

7. Bloch DA, Michel BA, Hunter GG, et al. The American College of Rheumatology. Development of classification and response criteria for the classification of vasculitis, patients and methods. Arthritis Rheum. 1990;2990(33):1068-73.

8. Jennette JC, Falk RJ, Bacon PA, et al. 2012 revised international Chapel Hill consensus conference nomenclature of vasculitides. Arthris Rheuma. 2013;65:1-11.

9. Luqmani RA, Suppiah R, Grayson PC, et al. Nomenclature and classification of vasculitis - update on the ACR/EULAR diagnosis and classification of vasculitis study (DCVAS). Clin Exp Immunol. 2011;164(suppl1):11-3.

10. Töpel I, Zorger N, Steinbauer M. Inflammatory diseases of the aorta. Gefässchirurgie 2016;21(Suppl 2):S80-6.

11. Dillon MJ, Eleftheriou D, Brogan P. Medium-size-vessel vasculitis. Pediatr Nephrol 2010;25(9):1641-52.

12. Wojciechowska J, Krajewski W, Krajewski $P$, et al. Granulomatosis with polyangiitis in otolaryngology. Clin Experimen Otor. 2016;9(1):8-13.

13. Benallegue $N$, Lozach $P$, Belizna $C$, et al. Acute coronary vasospasm in a patient with eosinophilic granulomatosis with polyangitis following NSAID administration: A case report. Medicine (Baltimore). 2016;95(47): e5259.

14. García-Nava M, Mateos-Toledo H, Guevara-Canseco AP, et al. Early interstitial lung disease in microscopic polyangiitis: case report and literature review. Reumatol Clin. 2016;2:S1699-258X(16)30132-2.

15. Hong J, Yang HR, Laboratory markers indicating gastrointestinal involvement of Henoch-Schönlein purpura in children. Pediatr Gastroenterol Hepatol Nutr. 2015;18(1):39-47. 
16. Monjazeb S, Philips RC, Wilkerson M. A case of leukocytoclastic vasculitis following influenza vaccination. JAAD Case Rep. 2016;17(4): 340-2.

17. Ozen S, Ruperto N, Dillon MJ, et al. EULAR/PReS endorsed consensus criteria for the classification of childhood vasculitides. Ann Rheum Dis. 2006;65:936-41.

18. Diplomatico M, Gicchino MF, Ametrano O, et al. A case of urticaria vasculitis in a female patient with lupus: Mycoplasma pneumoniae infection or lupus reactivation? Rheumatol Int. 2017;37(5):837-40.

19. Sáenz de Santa María M, Morales-Cabeza C, Noguerado-Mellado B, et al. Cutaneous leukocytoclastic vasculitis due to amoxicillin hypersensitivity. Ann Allergy Asthma Immunol. 2016;117(4):446-7.

20. Lee SH, Kim JH, Park S, et al. Pulmonary leukocytoclastic vasculitis as an initial presentation of myelodysplastic syndrome. Tuberc Respir Dis. 2016;79(4):302-6.

21. Ruelas Villavicencio AL, García Hidalho L, Reyes Gutiérrez E, et al. Causas de vasculitis cutánea en 59 pacientes mexicanos. Experiencia de un centro de tercer nivel. Dermatología Rev Mex. 2011:55(6):334-1.

22. Carlson A. The histological assessment of cutaneous vasculitis. Histopathology. 2010;56:3-23.

23. Pulido-Pérez A, Avilés-Izquierdo JA Suárez-Fernández R. Vasculitis cutáneas. Actas Dermosiiliogr. 2012;103(3):179-91.

24. Carlson A, Ng BT, Chen KR. Cutaneous vasculitis update: Diagnostic criteria classification, epidemiology, etiology, pathogenesis, evaluation and prognosis. Am J Dermatopathol. 2005;27:504-28.

25. Fiorentino D. Cutaneous vasculitis. J Am Acad Dermatol. 2003; 48(3): 311-40.

26. Al-Mutairi N. Spectrum of cutaneous vasculitis in adult patients from the Fawiniya region of Kuwait. Med Princ Pract. 2008;17(1):43-8.

27. Gibson L. Cutaneous vasculitis update. Dermatol Clin. 2001;19(4):603-15.
28. Kawakami T, Yamazaki M, Mizoguchi M, et al. High titer of serum antiphospholipid antibody levels in adult Henoch-Schönlein purpura and cutaneous leukocytoclastic angiitis. Arthritis Rheum. 2008;59(4):561-7.

29. Davin JC, Weening J. Diagnosis of Henoch-Schönlein purpura: renal or skin biopsy? Ped Nephrol. 2003;1201-3.

30. Oh $\mathrm{C}$, Lee $\mathrm{SH}, \mathrm{Heo} \mathrm{CP}$. A case suggesting lymphocytic vasculitis as a presenting sign of early undifferenciated connective tissue disease. Am Dermatopathol. 2003;25(5):423-7.

31. Monach PA. Biomarkers in Vasculitis. Curr Opin Rheumatol. 2014;26(1): 24-30.

32. Wiik A. Rational use of ANCA in the diagnosis of vasculitis. Rheumatol. 2002;41:481-3.

33. Mohan N, Kerr G. Diagnosis of vasculitis. Best Pact Research Clin Rheumatol. 2001;15(2):203-23.

34. Barnadas M, Perez E, Gich I. Diagnostic, prognostic and pathogenic value of the direct immunofluorescence test in cutaneous leucocytoclastic vasculitis. Int J Dermatol. 2004;43:19-26.

35. Fervenza F. Henoch-Schönlein purpura nephritis. Int J Dermatol. 2003;42:170-7.

36. Crowson A, Magro C, Usmani A, et al. Immunoglobulin A-associated lymphocytic vasculopathy: a clinicopathologic study of eight patients. J Cutan Pathol. 2002;29:596-601.

37. Sano $H$, Izumida $M$, Shimizu $H$. Risk factors of renal involvement and significant proteinuria in Henoch-Schönlein purpura. Eur J Pediatr. 2002:196-201.

38. Davin JC, Weening J. Henoch-Schönlein purpura: an update. Eur J Pediatr. 2001:689-95.

39. Craven A, Robson J, Ponte C, et al. ACR/EULAR-endorsed study to develop Diagnostic and Classification Criteria for Vasculitis (DCVAS). Clin Exp Nephrol. 2013;17:619-21. 